\title{
Performance prediction of massively parallel computation by Bayesian inference
}

\author{
Hisashi Kohashi $^{1}$, Harumichi Iwamoto ${ }^{1}$, Takeshi Fukaya ${ }^{2}$, Yusaku Yamamoto ${ }^{3}$ and Takeo Hoshi ${ }^{1 *}$ \\ ${ }^{1}$ Department of Mechanical and Physical Engineering, Tottori University, 4-101 Koyama-cho \\ Minami, Tottori 680-8552, Japan \\ ${ }^{2}$ Information Initiative Center, Hokkaido University, Kita 11 Nishi 5, Kita-ku, Sapporo 060- \\ 0811, Japan \\ ${ }^{3}$ Department of Communication Engineering and Informatics, The University of Electro- \\ Communications, 1-5-1 Chofugaoka, Chofu 182-8585, Japan \\ *Corresponding author: hoshi@tottori-u.ac.jp
}

Received November 05, 2021, Accepted December 09, 2021

\begin{abstract}
A performance prediction method for massively parallel computation is proposed. The method is based on performance modeling and Bayesian inference to predict elapsed time $T$ as a function of the number of used nodes $P(T=T(P))$. The focus is on extrapolation for larger values of $P$ from the perspective of application researchers. The proposed method has several improvements over the method developed in a previous paper, and application to realsymmetric generalized eigenvalue problem shows promising prediction results. The method is generalizable and applicable to many other computations.
\end{abstract}

Keywords parallel algorithm, Bayesian inference, performance modeling, generalized eigenvalue problem, Monte Carlo method

Research Activity Group Algorithms for Matrix / Eigenvalue Problems and their Applications

\section{Introduction}

A number of parallel algorithms for modern supercomputers have been developed in recent decades. However, it remains difficult for the general application researcher to choose the proper algorithm and/or set the values of tuning parameters that influence computational performance. One practical remedy is to predict the elapsed time $T$ as a function of the number of processor nodes $P(T=T(P))$. In particular, application researchers are interested in extrapolation for larger numbers of nodes.

The present paper proposes a performance prediction method featuring improvements over the method suggested in our previous paper [1]. In [1], we presented several generic performance models for the realsymmetric generalized eigenvalue problem in which the performance model function $T=T(P)$ is based on the applied algorithm and architecture and contains several fitting parameters. The parameters are estimated by Bayesian inference, whereby the elapsed time is predicted or extrapolated from the teacher data or the existing benchmark data. The method in the present paper improves that proposed in [1] in two respects: (i) it improves the performance model and the cost function for the Bayesian inference and (ii) it allows for a systematic comparison among different models and different teacher data set. Here, we use the same performance data as used in [1], since it shows the typical performance behavior of parallel computation and will enable the reader to compare the present results with those in [1].

The method and results are described in Sections 2 and 3, respectively. A summary is provided in Section 4.

\section{Method}

\subsection{Performance models}

Performance models were investigated in papers such as [2-9]. Our previous paper [1] proposed several generic performance models in which the elapsed time $T$ of a routine is decomposed into the following five terms

$$
\begin{aligned}
& T_{1}(P) \equiv \frac{c_{1}}{P}, \\
& T_{2}(P) \equiv c_{2}, \\
& T_{3}(P) \equiv c_{3} \log P, \\
& T_{4}(P) \equiv c_{4} \frac{\log P}{\sqrt{P}}, \\
& T_{5}(P) \equiv \frac{c_{5}}{P^{2}},
\end{aligned}
$$

where the parameters $\left\{c_{i}\right\}_{i}$ are to be estimated under the non-negative constraint $\left(\left\{c_{i} \geq 0\right\}_{i}\right)$, since each term is part of the elapsed time $\left(\left\{T_{i} \geq 0\right\}_{i}\right)$. The terms $T_{1}$ and $T_{2}$ represent the times of ideally parallel and non-parallel computations, respectively. The two-term model $T=$ $T_{1}+T_{2}$ is the famous Amdahl's law [10]. The term $T_{3}$ represents the setup time of MPI communications $[1,11]$. The three-term model $T=\sum_{i}^{3} T_{i}$ is a minimal model that has its minimum at $P=P^{*} \equiv \operatorname{argmin}_{P} T(P)$. The term $T_{4}(\propto \log P / \sqrt{P})$ expresses the time of MPI communications for matrix computation [1] and the term $T_{5}\left(\propto P^{-2}\right)$ is a phenomenological term introduced to express the 'super-linear' behavior in which the time decays faster than $T_{1}\left(\propto P^{-1}\right)[1]$.

This paper proposes an additional term responsible for 
the deceleration effect, when the total number of processor cores exceeds the matrix size $M$. In this situation, if the computation is carried out in the one-dimensional parallelism, no further speedup is obtained, but the overhead arises, which we assume to be proportional to $P$. Let $n_{\text {core }}$ be the number of cores per node, such situation appears, when $P>P_{\mathrm{c}} \equiv M / n_{\text {core }}$. We introduce the function that represents the above deceleration effect as

$$
T_{6}(P) \equiv c_{6} \frac{P}{1+\exp \left(-\left(P-P_{\mathrm{c}}\right)\right)},
$$

where a sigmoid-type function $\left(1+\exp \left(-\left(P-P_{\mathrm{c}}\right)\right)\right)^{-1}$ is used as a 'smoothed' step function. The term vanishes $\left(T_{6}(P) \approx 0\right)$ for $P \ll P_{\mathrm{c}}$ and is an increasing function $\left(T_{6}(P) \approx c_{6} P\right)$ for $P \gg P_{\mathrm{c}}$.

\subsection{Bayesian inference}

The parameter set $\left\{c_{i}\right\}_{i=1, \nu}$ in the performance models $T=\sum_{i}^{\nu} T_{i}$ is estimated by Bayesian inference using the Monte Carlo (MC) method. The posterior probability distribution is given by $\pi(X \mid D) \propto \pi(D \mid X) \pi(X)$, where $D$ represents the teacher data set $\left\{P_{j}, T_{j}^{(\exp )}\right\}_{j}$ and $X \equiv\left(c_{1}, c_{2}, \ldots, c_{\nu}\right)$ is the parameter set to be estimated. The cost function $F$ is defined from the relative error as

$$
F \equiv \sum_{j} \frac{\left|T\left(P_{j}\right)-T_{j}^{(\exp )}\right|^{2}}{\left|T_{j}^{(\exp )}\right|^{2}}
$$

and the likelihood $\pi(D \mid X)$ is defined to be proportional to $\exp (-F / \tau)(\pi(D \mid X) \propto \exp (-F / \tau))$. The given parameter $\tau(>0)$ is a measure of the tolerable uncertainty. The non-negative constraint $\left(c_{i} \geq 0\right)$ is reduced to the uniform prior distribution in the region $\left[0, c_{i}^{(\max )}\right]$. The upper limit $c_{i}^{(\max )}$ is chosen to be sufficiently large so that the region of non-zero posterior distribution $(\pi(X \mid D)>0)$ is contained in the region $\left[0, c_{i}^{(\max )}\right]$. The cost function proposed here differs from that used in [1], as discussed in Section 3.2.

\subsection{Target problem and technical details}

We chose as our target problem the real-symmetric generalized eigenvalue problem using matrix data VCNT22500 from the ELSES matrix library [12,13], where the matrix size $M$ is $M=22500$. The matrix data stem from the electronic state calculation by the ELSES simulator $[14,15]$ of a vibrating carbon nanotube. The elapsed time data appear in the 'total' time of Table 2 in [1], which was measured on the $\mathrm{K}$ computer using ScaLAPACK. The data are available for various numbers of nodes $P$, including $P=4,16,64,256,1024,4096$ and 10,000. This data set was chosen because the elapsed time $T(P)$ has a minimum at $P=1024$, which is typical in parallel computation. Since $n_{\text {core }}=8$ and $M=22500$ in the present case, the critical node number $P_{\mathrm{c}}$ is $P_{\mathrm{c}}=22500 / 8=2812.5$.

The MC method was carried out with the replica exchange Monte Carlo (REMC) algorithm [16] implemented in software package 2DMAT [17-19]. The method is standard and the use of 2DMAT is not es-
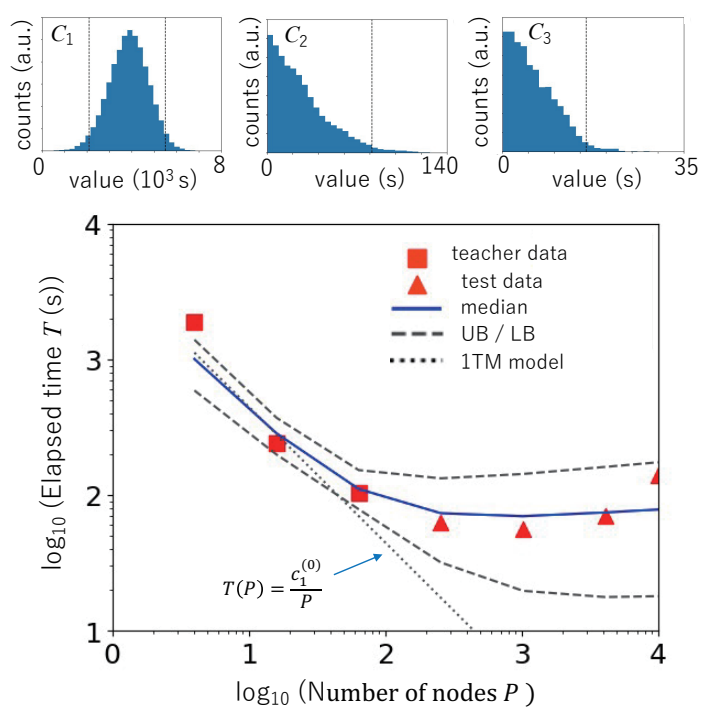

Fig. 1. Bayesian inference using the three-term model with three teacher data cases. Upper panels: The posterior probability distribution (histogram) for parameters $c_{1}$ (upper left panel), $c_{2}$ (upper middle panel), and $c_{3}$ (upper right panel). The upper and lower bounds of the $95 \%$ Highest Density Region (HDR) are drawn as vertical dashed lines in the upper left panel and the upper bounds of the HDR are drawn in the upper middle and right panels. Lower panel: The elapsed times $T(P)$ for experimental data and the Bayesian inference. The experimental data are shown for the three teacher data cases (squares) and the four test data cases (triangles). The results of the Bayesian inference are indicated by the median (solid line) and the upper and lower bounds (UB/LB) of the $95 \%$ HDR (dashed lines). The time by the roughly estimated one-term $(1 \mathrm{TM})$ model $\left(T=c_{1}^{(0)} / P\right)$ is also shown (dotted line).

sential. The REMC method uses multiple values of the tolerable uncertainty parameters $\tau$, the chosen values of which are $\tau=\tau_{0} \equiv 0.1, \tau_{1} \equiv 10^{2 / 3} \tau_{0}=0.464$, $\tau_{2} \equiv 10^{4 / 3} \tau_{0}=2.154, \tau_{3} \equiv 10^{2} \tau_{0}=10$. The total number of MCMC steps is $N_{\mathrm{MCMC}}=10^{6}$ for each value of the parameter $\tau$. The resultant sampling points with $\tau=0.1$ are used for the posterior probability density (histogram) except for the burn-in data of the early $N_{\text {MCMC }} / 2$ steps. The elapsed time for 2DMAT is approximately four minutes using a notebook computer.

\section{Results}

3.1 Results with different models and teacher data sets Fig. 1 shows the predictions by the three-term (minimal) model $\left(T=\sum_{i}^{3} T_{i}\right)$ with teacher data cases at $P=4,16,64$. The remainder of the experimental data, at $P=256,1024,4096,10000$, was used as the test data cases. The posterior probability density is shown in the histograms for $\left\{c_{i}\right\}_{i}=1,2,3$ in the left, middle and right panels, respectively, of the upper section of Fig. 1. The histograms for $c_{1}, c_{2}$ and $c_{3}$ show the maximums located near $c_{1} \approx c_{1}^{(0)} \equiv 4 \times 10^{3} \mathrm{~s}, c_{2} \approx 0$ and $c_{3} \approx 0$, respectively, which leads us to the one-term model $T \approx c_{1}^{(0)} / P$ as a rough estimation. The maximums for $c_{2}$ and $c_{3}$ are located near the origin, owing to the non-negative constraint $\left(\left\{c_{i} \geq 0\right\}_{i}\right)$. In the lower panel of Fig. 1, the elapsed times $T(P)$ of the experimental data are compared with those by the Bayesian inference. The exper- 
imental data are contained in the $95 \%$ HDR (Highest Density Region), except at $P=4$. The Bayesian inference indicates that parallelism with $P \geq 1000$ is not an efficient parallel computation, which offers a fruitful guideline for application researchers.

Fig. 2 shows a comparison of the Bayesian inference with different models and teacher data cases. Here, it is evident that the width of the $95 \% \mathrm{HDR}$ in the test data region is wider than in the teacher data region, as would be expected. In the lower panel of Fig. $2(\mathrm{a})$, for example, the widths of the $95 \% \mathrm{HDR}$ at $P=256,1024,4096,10000$ are wider than those at $P=4,16,64$. Figs. 2(a), (b) and (c) show the results of the three-term model $\left(T=\sum_{i}^{3} T_{i}\right)$, the four-term model $\left(T=\sum_{i}^{4} T_{i}\right)$ and the five-term model $\left(T=\sum_{i}^{5} T_{i}\right)$, respectively. The upper, middle and lower panels show the results with seven, five and three teacher data cases, respectively. Fig. 2(d) shows the results of the six-term model with seven and six teacher data cases in the upper and lower panels, respectively. It is noted that Fig. 2(d) does not include the results with the three and five teacher data cases, since the sigmoid-type function in $T_{6}$ is numerically zero $\left(\left(1+\exp \left(-\left(P-P_{\mathrm{c}}\right)\right)\right)^{-1} \approx\right.$ $0)$ in the three and five teacher data cases $(P=$ $4,16,64,256,1024)$, and these teacher data cases can not determine the coefficient $c_{6}$.

Figs. 2(a) and (b) indicate that the median curves in the three- and four-term models reproduce the experimental data satisfactorily, except at $P=4$ and $P=10000$. The deviations at $P=4$ and $P=10000$ stem from the limited representation ability of these models, since the deviations appear even with the seven teacher data cases in the upper panels of Figs. 2(a) and (b). The deviation at $P=4$ disappears with the fiveterm model, as shown Fig. 2(c), since the rapid decrease from $T(P=4)$ to $T(P=16)$ can be expressed by the super-linear term $\left(T_{5}\right)$. The deviation at $P=10000$ disappears with the six-term model and the six teacher data cases, as shown in Fig. 2(d), since the rapid increase from $T(P=4096)$ into $T(P=10000)$ can be expressed by the deceleration term $\left(T_{6}\right)$.

\subsection{Comparison of different cost functions}

We can now make a comparison of the different cost functions. In the previous paper [1], transformed variables $(\rho, \phi) \equiv(\log P, \log T)$ are used rather than the original variables $(P, T)$ and the cost function is defined as the absolute error of $F_{\text {trf }} \equiv \sum_{j}\left|\phi\left(\rho_{j}\right)-\phi_{j}^{(\exp )}\right|^{2}$, unlike in (7).

It is possible to compare the results produced by the different cost functions, since the results from the threeterm model with three teacher data cases are given in the lower panel of Fig. 2(a) and Fig. 6(a) of [1] and the results from the five-term model with three teacher data cases are given in the lower panel of Fig. 2(c) and Fig. 6(b) of [1]. The comparison suggests that the results using the present cost function $F$ are significantly better than those with the previous cost function $F_{\mathrm{trf}}$.

\subsection{Discussion}

It should be recalled that general application researchers are interested primarily in the location of the minimum point $\left(P=P^{*} \equiv \operatorname{argmin}_{P} T(P) \approx 10^{3}\right)$. In addition, application researchers have a solid need for a simple performance model in which the number of parameters is small and the parameters can be estimated from a small teacher data set without overfitting. Fig. 2 indicates that the three-term model with the three teacher data cases gives satisfactory prediction at $P \approx 10^{3}$, which leads to the conclusion that the minimal three-term model with three teacher data cases is effecctive.

A possible future direction for model development is prediction (extrapolation) with respect to both the number of nodes $P$ and the matrix size $M(T=T(P, M))$. The modeling will be fully realized, when the the coefficients $\left\{c_{i}\right\}$ in the term $\left\{T_{i}\right\}$ are re-defined as a function of $M\left(c_{i} \equiv c_{i}(M)\right)$. A reasonable modeling for matrix computation is a third order polynomial $\left(c_{i}(M) \equiv\right.$ $\left.c_{i 3} M^{3}+c_{i 2} M^{2}+c_{i 1} M+c_{i 0}\right)$. In such a case, the number of the parameters $\left(\left\{c_{i j}\right\}_{i j}\right)$ in the five-term model $(4 \times 5=20)$ is significantly larger than in the three-term model $(4 \times 3=12)$. The above discussion implies the importance of the three-term (simpler) model.

It would also be desirable to obtain more detailed performance data by measuring the elapsed time of lowerlevel routines. For example, one could measure the execution time of an MPI function, by inserting the timer into the source code. Such a detailed elapsed time could be used to construct a more accurate and reliable performance model for the entire solver.

\section{Summary}

A performance prediction method for parallel computations is proposed using parametrized performance models and Bayesian inference. The proposed method meets the need of application researchers, in particular, for performance extrapolation when the number of used nodes is large. Three-, four-, five-, and six-term performance models were proposed and applied to the realsymmetric generalized eigen-value problem. Comparison of the models indicates that the proposed method is effective even with the the combination of the three-term (minimal) model and the minimal teacher data set. Possible directions for further development of the method were suggested, including extrapolation with respect to both the number of nodes $P$ and the matrix size $M$. Importantly, the prediction method is general and can be used in applications other than the generalized eigenvalue problem.

\section{Acknowledgments}

The present research is supported by the Grant-in-Aid for Scientific Research (KAKENHI) from Japan Society for the Promotion of Science (19H04125, 20H00581) and JHPCN and HPCI in Japan (jh210044-NAH). 
(a)
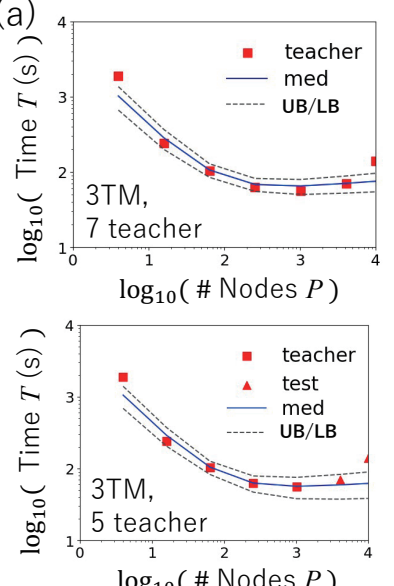

$\log _{10}$ ( \# Nodes P)

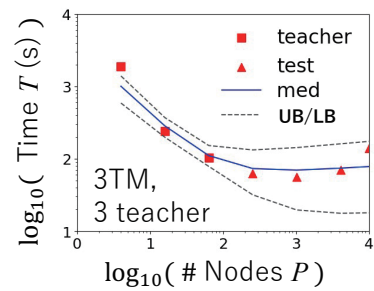

(b)

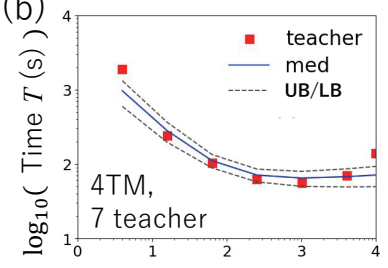

$\log _{10}($ \# Nodes $P$ )

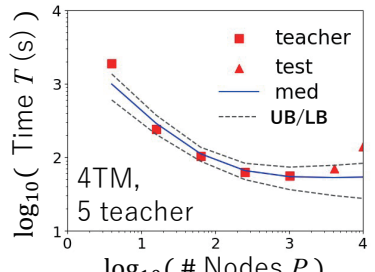

$\log _{10}($ \# Nodes $P$ )

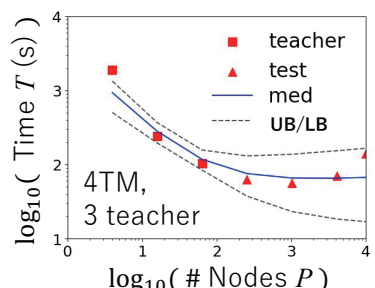

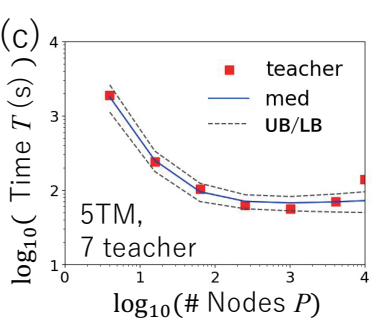

(d)
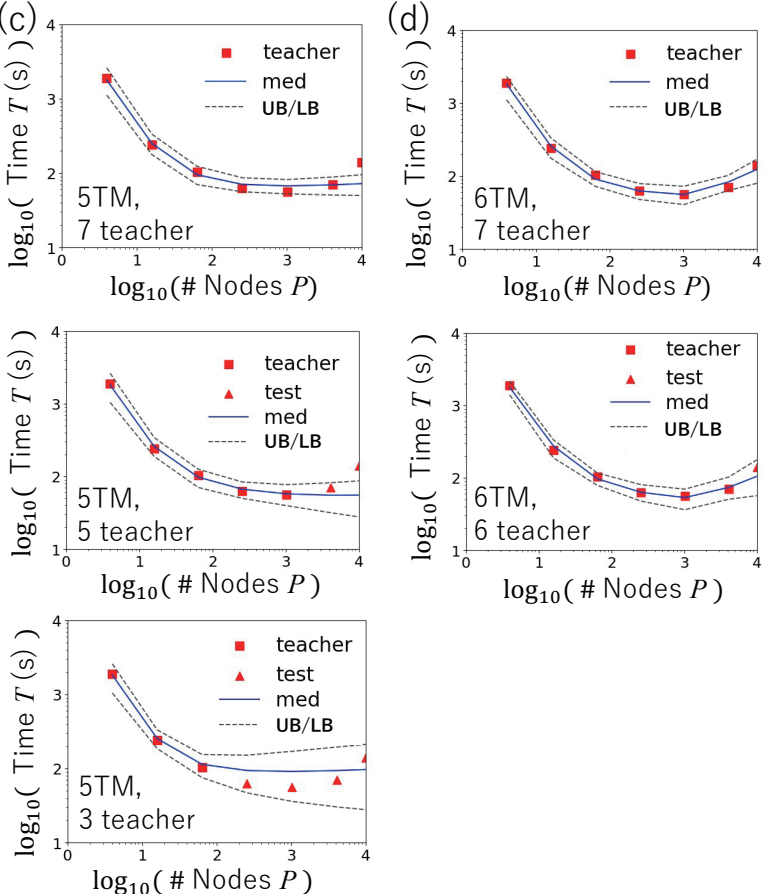

Fig. 2. Comparison of the elapsed times $T(P)$ for the experimental data and for the Bayesian inference. The experimental data are shown as squares for the teacher data cases and as triangles for the test data cases. The results of the Bayesian inference are indicated by the median (solid line) and the upper and lower bounds (UB/LB) of the $95 \%$ Highest Density Region (dashed lines). The Bayesian inference was carried out using (a) the three-term (3TM) model with the seven, five and three teacher data cases, (b) the four-term (4TM) model with the seven, five and three teacher data cases, (c) the five-term (5TM) model with the seven, five and three teacher data cases, and (d) the six-term (6TM) model with the seven and six teacher data cases.

\section{References}

[1] K. Tanaka et al., EigenKernel-a middleware for parallel generalized eigenvalue solvers to attain high scalability and usability, Jpn. J. Ind. Appl. Math., 36 (2019), 719-742.

[2] K. Dackland and B. Kågström, An hierarchical approach for performance analysis of scalapack-based routines using the distributed linear algebra machine, in: Applied Parallel Computing. Industrial Computation and Optimization, LNCS 1184, pp. 186-195, Springer, Berlin, 1996.

[3] J. Pješivac-Grbović et al., Performance analysis of MPI collective operation, Clust. Comput., 10 (2007), 127-143.

[4] T. Hoefler et al., Toward performance models of MPI implementations for understanding application scaling issues, in: Proc. Euro MPI Users' Group Meeting: Recent Advances in the Message Passing Interface, LNCS 6305, pp. 21-30, Springer, Berlin, 2010.

[5] E. Peise and P. Bientinesi, Performance modeling for dense linear algebra, in: 2012 SC Companion: High Performance Computing, Networking Storage and Analysis, pp. 406-416, IEEE Computer Society, 2012.

[6] P. Reisert et al., Following the blind seer-creating better performance models using less information, in: Proc. EuroPar 2017: Parallel Processing, 10417, pp.106-118, Springer, Berlin, 2017.

[7] T. Fukaya et al., Performance analysis of the Householdertype parallel tall-skinny QR factorizations toward automatic algorithm selection, in: Proc. VECPAR 2014: High Performance Computing for Computational Science, LNCS 8969, pp.269-283, Springer, Berlin, 2015.

[8] T. Fukaya et al., A case study on modeling the performance of dense matrix computation: Tridiagonalization in the EigenExa eigensolver on the K computer, in: Proc. 2018 IEEE International Parallel and Distributed Processing Symposium Workshop, pp. 1113-1122, IEEE Computer Society, 2018.

[9] A. Calotoiu et al., Extrapeak: Advanced automatic performance modeling for HPC applications, in: Software for Exascale Computing - SPPEXA 2016-2019, LNCSE 136, pp.
453-482, Springer, Cham, 2020.

[10] G. Amdahl, Validity of the single processor approach to achieving large-scale computing capabilities, in: AFIPS Conf. Proc., 30, pp. 483-485, AFIPS Press, Reston, 1967.

[11] P. S. Pacheco, Parallel Programming with MPI, Morgan Kaufmann, San Francisco, 1996.

[12] ELSES Matrix Library, http://www.elses.jp/matrix/. (accessed 15 Oct. 2021).

[13] T. Hoshi et al., Numerical aspect of large-scale electronic state calculation for flexible device material. Jpn. J. Ind. Appl. Math., 36 (2019), 685-698.

[14] ELSES, http://www.elses.jp/. (accessed 15 Oct. 2021).

[15] T. Hoshi et al., An order-N electronic structure theory with generalized eigenvalue equations and its application to a tenmillion-atom system, J. Phys. Condens. Matter, 24 (2012), 165502/1-6.

[16] K. Hukushima and K. Nemoto, Exchange Monte Carlo method and application to spin glass simulations, J. Phys. Soc. Jpn., 65, (1996), 1604-1608.

[17] 2DMAT, Ver. 1.0.1, https://www.pasums.issp.utokyo.ac.jp/2dmat/. (accessed 15 Oct. 2021).

[18] K. Tanaka et al., Development of data-analysis software for total-reflection high-energy positron diffraction (TRHEPD), Acta. Phys. Pol. A, 137 (2020), 188-192.

[19] T. Hoshi et al., Data-driven sensitivity analysis in surface structure determination using total-reflection high-energy positron diffraction (TRHEPD), Comp. Phys. Commun., 271 (2022), 108186 